\title{
ON THE COEFFICIENTS OF THE CYCLOTOMIC POLYNOMIAL
}

\author{
PAUL ERD ÖS
}

The cyclotomic polynomial $F_{n}(x)$ is defined as the polynomial whose roots are the primitive $n$th roots of unity. It is well known that

$$
F_{n}(x)=\prod_{d \mid n}\left(x^{n / d}-1\right)^{\mu(d)}
$$

For $n<105$ all coefficients of $F_{n}(x)$ are \pm 1 or 0 . For $n=105$, the coefficient 2 occurs for the first time. Denote by $A_{n}$ the greatest coefficient of $F_{n}(x)$ (in absolute value). Schur proved that $\lim \sup A_{n}=\infty$. Emma Lehmer ${ }^{1}$ proved that $A_{n}>c n^{1 / 3}$ for infinitely many $n$. In fact she proved that infinitely many such $n$ 's are of the form $p q r$ with $p, q$, and $r$ prime. In the present note we are going to prove that $A_{n}>n^{k}$ for every $k$ and infinitely many $n$. This is implied by the still sharper theorem:

THEOREM $1 .{ }^{2}$ For infinitely many $n$

$$
A_{n}>\exp \left[c_{1}(\log n)^{4 / 8}\right] .
$$

Specifically we may take $n=2 \cdot 3 \cdot 5 \cdots p_{b}$ for sufficiently large $k$.

Since

$$
\max _{|x|=1}\left|F_{n}(x)\right| \leqq A_{n}[\phi(n)+1],
$$

Theorem 1 follows at once from the following theorem.

THEOREM 2. For infinitely many $n$

$$
\max _{|x|=1}\left|F_{n}(x)\right|>\exp \left[c_{2}(\log n)^{4 / 3}\right] .
$$

For the proof of Theorem 2 we require several lemmas.

LEMma 1. Let $f(x)$ be a polynomial of highest coefficient 1 of degree $m$ with all its roots on the unit circle. Suppose that in ihe unit circle $f(x)$ assumes its maximum at $x_{0}\left(\left|x_{0}\right|=1\right)$, and let $y_{0}$ be the root of $f(x)$ closest to $x_{0}$. Then the arc between $x_{0}$ and $y_{0}$ is not less than $\pi / m$; and if it equals $\pi / m, f(x)=x^{m}-1$.

Received by the editors May 5, 1945, and, in revised form, August 22, 1945.

1 Bull. Amer. Math. Soc. vol. 42 (1936) p. 389. Reference to the older literature can be found in this paper.

2 Throughout the paper $c_{i}$ denotes a positive constant. 
This is a theorem of M. Riesz. ${ }^{3}$

Set $n=2 \cdot 3 \cdot 5 \cdots p_{k}$.

LEMMA 2. $p_{k} \sim \log n$.

LEMMA 3. $\phi(n) \sim e^{-\gamma} n / \log \log n$, where $\gamma$ is Euler's constant.

Lemma 2 is a well known consequence of the prime number theorem, and Lemma 3 follows from Lemma 2 and a theorem of Mertens. ${ }^{4}$

Lemma 4. Suppose $p_{k}{ }^{a} \leqq u \leqq p_{k}{ }^{4 / 3}$ where $1<a \leqq 4 / 3$, and let $N$ be the number of integers not greater than $u$ which are prime to $n$. Then for sufficiently large $k$,

$$
N>\left(1+c_{3}\right) u \phi(n) / n .
$$

PRoof. The integers in question are primes greater than $p_{k}$. By the prime number theorem

$$
N \sim u / \log u-p_{k} / \log p_{k} \sim u / \log u .
$$

Now $1 / \log u \geqq 3 /\left(4 \log p_{k}\right)$; and, by Lemmas 2 and $3, \log p_{k} \sim \log \log n$ $\sim e^{-\gamma} n / \phi(n)$. Lemma 4 now follows from $e^{-\gamma}<3 / 4$.

LEMMA 5. Suppose that for an infinite number of integers $m$ we are given a polynomial $g_{m}(x)$ of highest coefficient 1 of degree $m$, with all its roots on the unit circle and symmetric with respect to the real axis, and with $\left|g_{m}(1)\right|=1$, Let $t_{m}$ be a function of $m$ such that $t_{m} / m<\pi$ and $t_{m} \rightarrow \infty$ as $m \rightarrow \infty$. Suppose constants $c_{4}, \epsilon\left(0<\epsilon<1,0<c_{4}<1\right)$ given such that for any $u$ with $t_{m}{ }^{1-c} \leqq u \leqq t_{m}$ the number of roots of $g_{m}(x)=g_{m}\left(e^{i \theta}\right)$ with $|\theta| \leqq u / m$ is greater than $\left(1+c_{4}\right) u / \pi$, that is, greater than $\left(1+c_{4}\right)$ times the number of roots of $x^{m}=1$ in the same interval. Then for sufficiently large $m$

$$
\max _{|x|=1}|g(x)|>\exp \left(c_{5} t_{m}\right) .^{5}
$$

Proof. Denote by $A, B, C$ the following arcs:

$$
\begin{aligned}
& A:|\theta| \leqq t_{m}^{1-\epsilon} / m, \\
& B:|\theta| \leqq t_{m} / m, \\
& C:|\theta| \leqq\left(t_{m}+\pi\right) / m .
\end{aligned}
$$

We define new polynomials $h_{m}(x)=x^{m}+\cdots$ as follows. Outside $B$,

3 Jber. Deutschen Math. Verein. vol. 23 (1914) pp. 354-368.

4 See, for example, Hardy and Wright, Introduction to the theory of numbers, p. 349.

- An analogous but weaker theorem has been stated in a previous paper (Ann. of Math. vol. 44 (1943) p. 337. 
$h_{m}$ and $g_{m}$ have the same roots. In $A, h_{m}$ has no roots. On $B-A$ we place consecutive roots spaced by the angle $2 \pi / m$. Finally the remaining roots of $h_{m}$ are placed at the end points of $B$, half at each.

Let $\theta_{1}, \theta_{2}, \cdots$ and $\phi_{1}, \phi_{2}, \cdots$ denote the arguments of the roots of $g_{m}$ and $h_{m}$ in $B$ above the real axis; we number them in increasing order of magnitude. Our construction implies

$$
\phi_{r} \geqq \min \left(t_{m}^{1-\epsilon} / m+2 \pi r / m, t_{m} / m\right)
$$

while the hypothesis of Lemma 5 translates into

$$
\theta_{r} \leqq \max \left(t_{m}^{1-\epsilon} / m, 2 \pi r /\left(1+c_{4}\right) m\right) .
$$

From (1) and (2) we deduce $\phi_{r} \geqq \theta_{r}$, that is, the process has pushed roots of $g_{m}$ away from 1 . If $e^{i \theta}, e^{i \alpha}$ are points above the real axis respectively inside and outside $B$, then

$$
\partial\left|\left(e^{i \alpha}-e^{i \theta}\right)\left(e^{i \alpha}-e^{-i \theta}\right)\right| / \partial \theta=8 \sin \theta(\cos \alpha-\cos \theta)<0
$$

so that the process reduces $g_{m}$ outside $B$, that is,

$$
\left|h_{m}(x)\right| \leqq\left|g_{m}(x)\right|
$$

outside $B$.

We shall next prove

$$
\left|h_{m}(1)\right|>\exp \left(c_{6} t_{m}\right) .
$$

Take $m$ large enough so that $t_{m}^{e} \geqq 2$ and confine $r$ to the interval

$$
\begin{aligned}
\left(1+c_{4}\right) t_{m}^{1-\epsilon} / 2 \pi & \leqq r \\
& \leqq\left(1+c_{4}\right) t_{m} / 4 \pi .
\end{aligned}
$$

Then (2) reduces to

$$
\theta_{r} \leqq 2 \pi r /\left(1+c_{4}\right) m .
$$

Since from (5) and $c_{4}<1$ we have $2 \pi r \leqq t_{m}$, (1) similarly becomes

$$
\phi_{r} \geqq 2 \pi r / m \text {. }
$$

Combining $\left(1^{\prime}\right)$ and $\left(2^{\prime}\right)$ we find $\phi_{r} / \theta_{r}-1 \geqq c_{4}$ whence

$$
\left|1-\exp \left(i \phi_{r}\right)\right| \geqq c_{7}\left(1-\exp \left(i \theta_{r}\right) \mid .\right.
$$

From this it follows that $\left|h_{m}(1)\right| \geqq c_{7}^{R}\left|g_{m}(1)\right|$, where $R$ is the number of values of $r$ permitted in (5). Since for large $m, R>c_{8} t_{m}$, we have $c_{7}^{R}>\exp \left(c_{6} t_{m}\right)$, proving (4).

Let $X$ denote the number of roots of $h_{m}$ at the end points of $B$. 
It follows from our hypothesis that $X>c_{4} t_{m} / \pi$. We define a further polynomial $k_{m}(x)=x^{m}+\cdots$ by placing roots at the points with arguments $\pm \pi / m, \pm 3 \pi / m, \pm 5 \pi / m, \cdots$ on the $\operatorname{arc} A$. If the number of these points is $Y$, then $Y<c_{9} t_{m}{ }^{1-}$. We place $(X-Y) / 2$ roots of $k_{m}$ at each end point of $B$ and otherwise the roots of $h_{m}$ and $k_{m}$,coincide.

In moving the $Y$ roots to pass from $h_{m}$ to $k_{m}$ the greatest migration along the arc is from $t_{m} / m$ to $\pi / m$. Hence

$$
\left|k_{m}(1)\right| \geqq\left(c_{10} / t_{m}\right)^{Y}\left|h_{m}(1)\right| \text {. }
$$

Outside the arc $C$ the movement of roots tends to increase $h_{m}$; the worst place is right at the end points of $C$ and there we have the similar estimate

$$
\left|k_{m}(x)\right| \leqq\left(c_{11} t_{m}\right)^{Y}\left|h_{m}(x)\right|
$$

outside $C$. Now $k_{m}$ has roots all through $B$ spaced $2 \pi / m$ apart, and $k_{m} \neq x^{m}-1$. By Lemma $1, k_{m}$ must assume its maximum at a point $x_{0}$ outside $C$. Then, applying (3), (7), (6), and (4) in succession, we obtain

$$
\begin{aligned}
\left|g_{m}\left(x_{0}\right)\right| & >\left(c_{11} t_{m}\right)^{-Y}\left(c_{10} / t_{m}\right)^{Y} \exp \left(c_{6} t_{m}\right) \\
& =\left(c_{12} / t_{m}\right)^{2 Y} \exp \left(c_{6} t_{m}\right) \\
& >\exp \left(c_{5} t_{m}\right),
\end{aligned}
$$

which completes the proof of Lemma 5 .

Proof of Theorem 2. Take $n=2 \cdot 3 \cdot 5 \cdots p_{k}$. It is well known that $\left|F_{n}(1)\right|=1$. In view of Lemma 4 , we may apply Lemma 5 with $m, g_{m}(x), t_{m}, \epsilon$ replaced by $\phi(n), F_{n}(x), p_{h}^{4 / 3}$ and $1 / 6$ respectively. The conclusion is precisely Theorem 2 .

Theorem 2 is probably not the best result. It should not be difficult to extend the method to show that

$$
A_{n}>\exp (\log n)^{k}
$$

for every $k$ and infinitely many $n$. A very much stronger result may be true, namely

$$
A_{n}>\exp \left(c_{13} n / \log \log n\right)
$$

for infinitely many $n$. If true, this would be essentially the best possible result, because for a certain $c_{14}$ and all $n$,

$$
A_{n}<\exp \left(c_{14} n / \log \log n\right) \text {. }
$$

(The proof is omitted.)

The possibility that (7) may be true is indicated in the following theorem. 
TheOREM 3. Let $n$ be the product of $k$ distinct primes $p_{1}, p_{2}, \cdots, p_{h}$ and denote by $f(x)$ the number of integers not greater than $x$ which are relatively prime to $n$. Let

$$
\begin{aligned}
P & =\left(1-1 / p_{1}\right) \cdots\left(1-1 / p_{k}\right), \\
g(x) & =f(x)-P x .
\end{aligned}
$$

Then there exists an $x_{0}, 1 \leqq x_{0}<n$, such that

$$
\left|g\left(x_{0}\right)\right|>c_{15} 2^{k / 2}(\log k)^{-1 / 2} .
$$

The connection between Theorem 3 and (8) is as follows. The function $g(x)$ measures how much the roots of $F_{n}(x)$ are displaced from the uniform distribution. Lemma 5 then suggests that it might be possible to prove

$$
\max _{|x|=1}\left|F_{n}(x)\right|>\exp \left[c_{16} 2^{k / 2}(\log k)^{-1 / 2}\right] .
$$

If in particular we take $n=2 \cdot 3 \cdot 5 \cdots p_{k}$, then

$$
p_{k} \sim \log n \sim k \log k,
$$

and (10) is a result similar to (8).

Proof of Theorem 3. The usual sieve process gives

$$
f(x)=[x]-\sum_{\left.p\right|_{n}}\left[\frac{x}{p}\right]+\sum_{p q \mid n}\left[\frac{x}{p q}\right]-\cdots=\sum_{r \mid n} \mu(r)[x / r] .
$$

Define $(x / r)=x / r-[x / r]$, so that $g(x)=\sum_{r \mid n} \mu(r)(x / r)$. Then

$$
\sum_{x=1}^{n}[g(x)]^{2}=\sum_{r, s \mid r, n} \mu(r) \mu(s) \sum_{x=1}^{n}(x / r)(x / s) .
$$

Let $r=u d, s=v d,(u, v)=1$. Then the final sum becomes

$$
\begin{aligned}
\sum_{x=1}^{n}(x / r)(x / s)= & n d(r s)^{-2} \sum_{a=0}^{d-1}[a+a+d+\cdots+a+(u-1) d] \\
& \cdot[a+a+d+\cdots+a+(v-1) d] \\
= & n\left(3 r s-3 r-3 s+d^{2}+2\right) / 12 r s .
\end{aligned}
$$

In carrying out the second summation, the first three terms vanish. Hence

$$
\begin{aligned}
12 \sum_{x=1}^{n}[g(x)]^{2} & =n \sum_{r, s \mid n}\left(d^{2}+2\right) \mu(r) \mu(s) / r s \\
& =n\left(2^{k} P+2 P^{2}\right) .
\end{aligned}
$$


Now $P>c_{17} / \log k$, as follows a fortiori from Lemma 3. Hence

$$
\sum_{x=1}^{n}[g(x)]^{2}>c_{18} n 2^{k} / \log k,
$$

from which the existence of an $x_{0}$ satisfying (9) follows at once.

I am indebted to Dr. Irving Kaplansky who shortened some of the proofs and extensively revised the first draft of the manuscript.

UNIVERSITY OF MiCHIGAN 